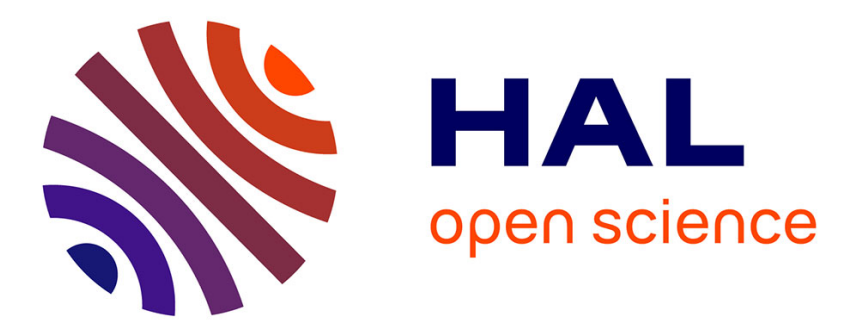

\title{
Comparison of observer approaches for actuator fault estimation in semi-active suspension systems
}

Manh Quan Nguyen, Olivier Sename, Luc Dugard

\section{To cite this version:}

Manh Quan Nguyen, Olivier Sename, Luc Dugard. Comparison of observer approaches for actuator fault estimation in semi-active suspension systems. SysTol 2016 - 3rd International Conference on Control and Fault-Tolerant Systems, Sep 2016, Barcelone, Spain. hal-01361742

\section{HAL Id: hal-01361742 \\ https://hal.science/hal-01361742}

Submitted on 7 Sep 2016

HAL is a multi-disciplinary open access archive for the deposit and dissemination of scientific research documents, whether they are published or not. The documents may come from teaching and research institutions in France or abroad, or from public or private research centers.
L'archive ouverte pluridisciplinaire HAL, est destinée au dépôt et à la diffusion de documents scientifiques de niveau recherche, publiés ou non, émanant des établissements d'enseignement et de recherche français ou étrangers, des laboratoires publics ou privés. 


\title{
Comparison of observer approaches for actuator fault estimation in semi-active suspension systems
}

\author{
M.Q.Nguyen ${ }^{1 *}$, O.Sename ${ }^{1}$, L.Dugard ${ }^{1}$
}

\begin{abstract}
In this paper, the actuator fault estimation problem of semi-active suspension systems is considered. For instance, an oil leakage in the damper could cause a reduction of the damping force. The fault estimation requires a modeling of the damper fault (both multiplicative and additive fault models can be used). Three observer-based approaches are compared for fault estimation: an observer using fast adaptive fault estimation (FAFE) approach (used for estimation of additive faults), a parametric adaptive observer (AO) and a switched LPV observer (LPVO) (both intended to estimate mulplicative faults); Since the damper fault estimation is strongly affected by the unknown road disturbances, an $H_{\infty}$ performance objective is used to reduce the effect of disturbances on the estimation error for performance assessment. Some simulations are performed on a quarter car model to validate these methodologies and a comparison is then given to shows the interest of each method.
\end{abstract}

Keywords: Fault estimation, semi-active damper fault, adaptive observer, fast fault adaptive estimation, LPV observer.

\section{INTRODUCTION}

Fault Detection and Isolation (FDI) has received significant interest in both research and application domains since the last two decades. For complex systems highly equipped with sensors and actuators such as automotive applications, FDI has become one essential issue for reliability and safety. The design of a FDI procedure includes fault detection, isolation and estimation. Among them, fault estimation is a key step in designing a fault tolerant control. Many different approaches to estimate a fault which can be either actuator or sensor malfunction. Let us mention some classical methods, based on the parity space theory [1] to generate the residues and approximate the fault, or the bank of observers approach [2], or the sliding mode observers [3]. Recently, a new approach in [4] considered the fault element as a state of the augmented system and designed an extended observer to estimate, at the same time, the state and the fault of system. However, it is limited to constant faults $(\dot{f}(t)=0)$. Then, [5] presented a method allowing to evaluate the timevarying fault by using a fast adaptive fault estimation (FAFE) methodology based on an adaptive observer. However, the authors solved the problem with a regular LTI system without considering the disturbances. Recently, [6] proposed an adaptive polytopic unknow input observer for time-varying fault estimation, for a class of descriptor LPV systems. Then, several works have been done for fault estimation and fault

\footnotetext{
1 Univ. Grenoble Alpes, GIPSA-lab, F-38000 Grenoble, France CNRS, GIPSA-lab, F-38000 Grenoble, France. $\quad$ manh-quan.nguyen, olivier.sename, luc.dugard\}@gipsa-lab.grenoble-inp.fr
}

tolerant control for LPV systems, such as LPV observer design for state and fault estimation in [7], [8], interval observer for LPV systems in [9], LPV sliding mode observer with fault compensation in [10], virtual sensor approaches in [11].

In the automotive field, the demand is now concerned by safer and more comfortable vehicles. Among all sub-systems impacting the vertical vehicle dynamics, the supension systems play a key role since they ensure the link between the wheels and the chassis (see [12]). Suspension systems are efficient actuators to considerably improve not only passengers comfort but also car road holding [13]. Therefore, the development of intelligent suspension systems has gained a lot of attention during last years. In particular, semi-active suspensions are today more and more used because of their efficiency, while being less expensive and comsumming less energy than pure active suspensions. These systems consist of a shock absorber which is able to online change its damping characteristics to enhance the vehicle dynamics.

While the semi-active suspensions have big advantages in enhancing vehicle dynamics, the necessity of fault diagnosis for these systems is more and more present. In particular, it has been noted that semi-active dampers are more susceptible to faults than their traditional counterpart. Therein, oil leakage is the most common fault, and its effect is a reduction of the damping force. The estimation of suspension faults is an indispensable step in the Fault Tolerant Control (FTC) to prevent handling and comfort deterioration. [14], [15] proposed a LPV/FTC scheduled by fault estimation which is based on the parity space theory and fast adaptive observer approaches respectively. Recently, [16] presented various methods using an observer-based approach and a parameter identification approach to estimate damper fault.

This paper aims at comparing three methodologies to deal with the actuator fault estimation problem of the semi-active suspension systems in the framework of observer based approaches. Note that such a comparison is proposed here for the first time in the case of suspension system. In the first method, the fault is represented as an additive fault, then a fast fault adaptive estimation approach (FAFE approach) is used for fault estimation. In the latter approaches, the damper fault is modeled in a multiplicative way using a effectiveness coefficient. Then, this coefficient can be estimated by a parametric adaptive observer (AO approach) and a switched LPV observer (LPVO approach). The damper fault estimation is strongly affected by the unknown road disturbances. To deal with this problem, an $H_{\infty}$ performance objective is used to reduce the effect of disturbances on the estimation error 
for performance assement. The comparison between three methods is given thereafter.

The paper is organized as follows. Section II presents the vehicle modeling and problem statement. Section III, IV and $\mathrm{V}$ present the different methods allowing to estimate the damper fault using FAFE, AO and LPVO approaches respectively. In section VI, some simulation results are given. Finally, some conclusions are drawn in the last section.

Notations: $P^{T}$ is the transposed of matrix $P, 0$ and $I$ denote zeros and identity matrix of appropriate dimensions, the star symbol $\star$ in a symmetric matrix denotes the transposed block in the symmetric position.

\section{PRoblem STATEMENT}

The dynamic behavior of a quarter car model with a semiactive suspension, taken into account in this work, is depicted as in Fig.1:

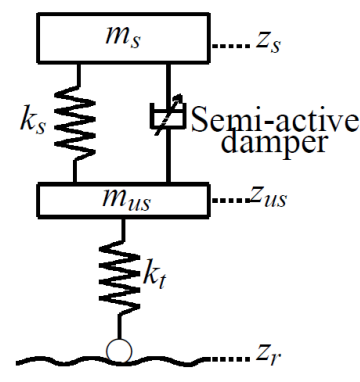

Fig. 1. Quarter-car vehicle model

In this model, the quarter vehicle body is represented by the sprung mass $\left(m_{s}\right)$, the wheel and tire are represented by the unsprung mass $\left(m_{u s}\right)$. They are connected by a spring with the stiffness coefficient $k_{s}$ and a semi-active damper. The tire is modeled by a spring with the constant stiffness coefficient $k_{t}$. As seen in the figure, $z_{s}$ (respectively $z_{u s}$ ) is the vertical displacement around the equilibrium point of $m_{s}$ (respectively $m_{u s}$ ) and $z_{r}$ stands for the road disturbances.

The dynamical equations are governed by:

$$
\left\{\begin{array}{l}
m_{s} \ddot{z}_{s}=-k_{s}\left(z_{s}-z_{u s}\right)-F_{s a} \\
m_{u s} \ddot{z}_{u s}=k_{s}\left(z_{s}-z_{u s}\right)+F_{s a}-k_{t}\left(z_{u s}-z_{r}\right)
\end{array}\right.
$$

where $F_{s a}$ is the semi-active damper force, which can be represented by a linear model:

$$
F_{s a}=c \dot{z}_{d e f}
$$

where $c$ is the damping coefficient that must satisfy the dissipativity constraint $c_{\text {min }} \leq c \leq c_{\text {max }}$.

Let us assume now that a fault occurs on the semi-active damper e.g an oil leakage which induces the effectiveness loss of the damper. Denoting $\alpha$ the efficiency coefficient of the damper, and $\bar{F}_{s a}(t)$ stands for the force of the faulty damper, then one has the following relation:

$$
\bar{F}_{s a}(t)=(1-\alpha) F_{s a}(t)
$$

In this study, $\alpha$ is considered as a constant parameter. For example $\alpha=0$ corresponds to a healthy damper, $\alpha=0.8$ corresponds to that the damper losses $80 \%$ its efficency. $\alpha=$ 1 represents a completely damper failure. Note that (3) is multiplicative fault modeling, but an additive fault modeling could be also used (see section III).

The problem consists now in estimating the damper fault, i.e the coefficient $\alpha$ which could be estimated by several approaches: a fast adaptive fault estimation (FAFE) approach (where an additive fault modeling is used, see section III), an adaptive observer (section IV) and a switched LPV observer (section $\mathrm{V}$ ).

\section{METHOD 1: FAFE APPROACH}

In this section, an addittive modeling for damper fault is considered. In fact, let us decompose the total semiactive damper force as: $F_{s a}(t)=c_{0} \dot{z}_{d e f}(t)+u(t)$, where $c_{0}$ is damping coefficient for the passive damper, $\dot{z}_{d e f}$ is deflection speed and $u(t)$ is control input. Then, the faulty damper force in (3) can be rewritten as follows:

$$
\bar{F}_{s a}(t)=(1-\alpha) F_{s a}(t)=c_{0} \dot{z}_{d e f}(t)+u(t)+f(t)
$$

where $f(t)$ is the loss of damper force and given by $f(t)=$ $-\alpha\left(c_{0} \dot{z}_{\text {def }}(t)+u(t)\right)$. Note that the effectiveness coefficient $\alpha$ is slowly varing or constant, but the additive fault $f=$ $-\alpha\left(c_{0} \dot{z}_{\text {def }}+u\right)$ could be time varying even if $\alpha$ is constant.

Now, the state space representation of quarter car model (1) while considering a faulty semi-active damper is given as follows:

$$
\begin{aligned}
\dot{x}(t) & =A x(t)+B_{1} w(t)+B_{2} u(t)+E f(t) \\
y(t) & =C x(t)
\end{aligned}
$$

where $x=\left(z_{s}, \dot{z}_{s}, z_{u s}, \dot{z}_{s}\right)^{T} \in R^{n}$ is the state vector, $w=z_{r}$ is the disturbance input, $u \in R^{m}$ is the control input, $f$ stand for damper fault. $y=\left[z_{\text {def }}, \dot{z}_{\text {def }}, z_{s}\right] \in R^{p}$ is the output vector. Such a choice of $y$ allows to satisfy Assumption 1 presented later.

$$
\begin{aligned}
& A=\left[\begin{array}{cccc}
0 & 1 & 0 & 0 \\
\frac{-k_{s}}{m_{s}} & \frac{-c_{0}}{m_{s}} & \frac{k_{s}}{m_{s}} & \frac{c_{0}}{m_{s}} \\
0 & 0 & 0 & 1 \\
\frac{k_{s}}{m_{u s}} & \frac{c_{0}}{m_{u s}} & -\frac{k_{s}+k_{t}}{m_{u s}} & -\frac{c_{0}}{m_{u s}}
\end{array}\right], B_{1}=\left[\begin{array}{c}
0 \\
0 \\
0 \\
\frac{k_{t}}{m_{u s}}
\end{array}\right], \\
& B_{2}=\left[\begin{array}{cccc}
0 & \frac{-1}{m_{s}} & 0 & \frac{1}{m_{u s}}
\end{array}\right]^{T}, E=\left[\begin{array}{cccc}
0 & \frac{-1}{m_{s}} & 0 & \frac{1}{m_{u s}}
\end{array}\right]^{T} \text {, } \\
& C=\left[\begin{array}{cccc}
1 & 0 & -1 & 0 \\
0 & 1 & 0 & -1 \\
1 & 0 & 0 & 0
\end{array}\right]
\end{aligned}
$$

The fault estimation problem consists now in estimating $\hat{f}(t)$, and the estimation of $\alpha$ can be given by: $\hat{\alpha}=\hat{f} / F_{s a}$.

Since $f(t)$ can be fast time varying, so in order to get a good estimation of the damper fault, the Fast Adaptive Fault Estimation (FAFE) approach proposed in [5], [6] is used. Moreover, since the vehicle dynamic is affected by the road disturbance, in order to improve the estimation quality, some extended results using an $H_{\infty}$ performance is used to attenuate the disturbance effects on the state and fault estimation error. 
Let us consider now the state space repesentation (5) with additive fault $f$. The following assumptions are satisfied:

- Assumption 1: rank $(\mathrm{CE})=\operatorname{rank}(\mathrm{E})$, and the invariant zeros of (A,E,C) are on the left half plane.

- Assumption 2: The fault $f(t)$ and its time derivative are norm bounded i.e: $0 \leq\|f(t)\|<\alpha_{1}$ and $0 \leq\|\dot{f}(t)\|<\alpha_{2}$ with $0 \leq \alpha_{1}, \alpha_{2}<\infty$.

The state space repesentation (5) includes the road disturbance $w=z_{r}$ which is an unknown input disturbance in the suspension system. Therefore, while estimating the fault actuator $f(t)$, one needs to take into account the effect of this unknown input. To deal with this problem, an unknown input adaptive fault observer is proposed and given as follows:

$$
\begin{aligned}
\dot{z}(t) & =N z(t)+G u(t)+L y(t)+T_{1} E \hat{f}(t) \\
\hat{x}(t) & =z(t)+T_{2} y(t) \\
\hat{y}(t) & =C \hat{x}(t)
\end{aligned}
$$

where $z \in R^{n}$ is the state variables of the observer, $\hat{x} \in R^{n}$ the estimated state variables. $\hat{y} \in R^{p}$ is the estimated output vector and $\hat{f}(t) \in R^{r}$ is the estimation of the damper fault $f(t) . N, G, L, T_{1}, T_{2}$ are the observer matrices to be designed to enable the convergence of the state and fault.

Denote $e_{x}(t)=x(t)-\hat{x}(t), e_{y}(t)=y(t)-\hat{y}(t), e_{f}(t)=$ $f(t)-\hat{f}(t)$ as the state, output and fault estimation error respectively. Since there exists a full-row rank matrix $\left[\begin{array}{ll}T_{1} & T_{2}\end{array}\right]\left[\begin{array}{l}I \\ C\end{array}\right]=I$, i.e $T_{1}=I-T_{2} C$, then $e_{x}=\left(I-T_{2} C\right) x-z=$ $T_{1} x-z$ and

$$
\begin{aligned}
\dot{e}_{x} & =T_{1} \dot{x}-\dot{z} \\
& =T_{1}\left(A x+B_{1} w+B_{2} u+E f\right)-\left(N z+G u+L y+T_{1} E \hat{f}\right)
\end{aligned}
$$

After some manipulations, one has:

$$
\begin{aligned}
\dot{e}_{x} & =N e_{x}+T_{1} E e_{f}+ \\
& +\left(T_{1} A-N T_{1}-L C\right) x+\left(T_{1} B_{2}-G\right) u+T_{1} B_{1} w
\end{aligned}
$$

If the following conditions hold:

$$
T_{1} A-N T_{1}-L C=0, T_{1} B_{2}-G=0,
$$

then, the estimation error dynamic is given by:

$$
\begin{aligned}
& \dot{e}_{x}=N e_{x}+T_{1} E e_{f}+T_{1} B_{1} w \\
& e_{y}=C e_{x}
\end{aligned}
$$

Since $T_{1}=I-T_{2} C$, then by denoting $K=N T_{2}-L$, one has $N=T_{1} A+K C$.

The equation (7), governing the state and fault estimation errors, is affected by the unknown road disturbance $w$. If $T_{1} B_{1}=0$, the effect of the road disturbance on the estimated states is cancelled. This condition is equivalent to $\left(I-T_{2} C\right) B_{1}=0$, where $T_{2}$ has to be determined. This equation is solvable if and only if $\operatorname{rank}\left(C B_{1}\right)=\operatorname{rank}\left(B_{1}\right)$. Otherwise, the disturbance effect has to be minimized and the problem is to find $N$ such that $N$ is stable and the effect of $w$ on $e_{y}$ is minimized.

In order to minimize the disturbance effect on the estimation error, we use the Bounded Real Lemma for the $H_{\infty}$ performance index to design the observer.
Then, the following theorem gives an extension to the results in [5] and aims at minimizing the disturbance effect on the estimation error and ensuring the convergence of the estimation error in (7):

Theorem 1: Under the assumptions 1-2, given scalars $\sigma, \mu, \gamma_{2}>0$, if there exist symmetric positive definite matrices $Q, P_{1}$, and matrices $W, U$ such that the following conditions hold:

$$
\left[\begin{array}{ccc}
M_{11} & M_{12} & M_{13} \\
\star & M_{22} & M_{23} \\
\star & \star & M_{33}
\end{array}\right]<0
$$

and

$$
E^{T} T_{1}^{T} Q=U C
$$

where:

$$
\begin{aligned}
M_{11} & =\left(T_{1} A\right)^{T} Q+Q\left(T_{1} A\right)+C^{T} W^{T}+W C+C^{T} C \\
M_{12} & =-\frac{1}{\sigma}\left[\left(T_{1} A\right)^{T} Q+C^{T} W^{T}\right] T_{1} E \\
M_{13} & =Q T_{1} B_{1} \\
M_{22} & =-\frac{2}{\sigma} E^{T} T_{1}^{T} Q T_{1} E+\frac{1}{\sigma \mu} P_{1} \\
M_{23} & =-\frac{1}{\sigma} E^{T} T_{1}^{T} Q T_{1} B_{1} \\
M_{33} & =-\gamma_{2}^{2} I
\end{aligned}
$$

then, the following fault estimation algorithm:

$$
\dot{\hat{f}}(t)=\Gamma U\left(\dot{e}_{y}(t)+\sigma e_{y}(t)\right)
$$

can ensure that $e_{x}(t)$ and $e_{f}(t)$ are uniformly bounded, where $\Gamma \in R^{r \times r}$ is a symmetric positive definite learning rate matrix. Proof: Consider the following Lyapunov function:

$$
V\left(e_{x}, e_{f}\right)=e_{x}^{T}(t) Q e_{x}(t)+\frac{1}{\sigma} e_{f}^{T}(t) \Gamma^{-1} e_{f}(t)
$$

Then, the $L_{2}$ induced gain conditon $\left\|e_{y}\right\|_{2}<\gamma_{2}\|w\|_{2}$ is satisfied if:

$$
\dot{V}+e_{y}^{T} e_{y}-\gamma_{2}^{2} w^{T} w<0
$$

Thanks to the Bounded Real Lemma and following the same procedure as in [5], the proof can be infered easily $\square$.

Then, it is easy to show that the estimated fault can be deduced from the expression (10) as follows :

$$
\hat{f}(t)=\Gamma U\left(e_{y}(t)+\sigma \int_{t_{f}}^{t} e_{y}(\tau) d \tau\right)
$$

where $t_{f}$ is the time since fault occurs. The fault reconstruction in (13) combines a proportional term with an integral one that allows to improve the fault estimation speed.

Therefore, to estimate the fault, one needs to solve the 2 conditions in Theorem 1 which consists of a Linear Matrix Inequality (8) and an equality matrix constraint (9) which are transformed into the following optimization problem: Min $\gamma$ subjects to (8) and

$$
\left[\begin{array}{cc}
\gamma I & E^{T} Q-U C \\
\left(E^{T} Q-U C\right)^{T} & \gamma I
\end{array}\right]>0
$$


Solving this optimization problem, the observer matrices can be computed by: $N=T_{1} A+K C, K=Q^{-1} W$ and the estimation of the fault actuator is performed.

\section{Method 2: AdAPtive OBSERVER (AO) APPROACH}

This part shows the procedure to estimate a multiplicative damper fault. As section III, the total damper force is still written as: $F_{s a}(t)=c_{0} \dot{z}_{d e f}(t)+u(t)$, where $c_{0}$ is damping coefficient for the passive damper, $u(t)$ is control input. From (3), one has:

$$
\bar{F}_{s a}(t)=(1-\alpha) F_{s a}(t)=(1-\alpha)\left(c_{0} \dot{z}_{d e f}(t)+u(t)\right)
$$

Then the state space representation of the quarter model (1) under the damper fault is given by:

$$
\begin{aligned}
\dot{x}(t) & =A x(t)+B_{1} w(t)+B_{2} u(t)+\Psi(y, u, t) \alpha \\
y(t) & =C x(t)
\end{aligned}
$$

where $x=\left(z_{s}, \dot{z}_{s}, z_{u s}, \dot{z}_{s}\right)^{T} \in R^{n}$ is the state vector, $w=z_{r}$ is the disturbance input, $u \in R^{m}$ is the control input, $\alpha$ stand for damper fault. $y=\left[z_{\text {def }}, \dot{z}_{d e f}, z_{s}\right] \in R^{p}$ is the output vector,.

$$
\begin{gathered}
A=\left[\begin{array}{cccc}
0 & 1 & 0 & 0 \\
\frac{-k_{s}}{m_{s}} & \frac{-c_{0}}{m_{s}} & \frac{k_{s}}{m_{s}} & \frac{c_{0}}{m_{s}} \\
0 & 0 & 0 & 1 \\
\frac{k_{s}}{m_{u s}} & \frac{c_{0}}{m_{u s}} & -\frac{k_{s}+k_{t}}{m_{u s}} & -\frac{c_{0}}{m_{u s}}
\end{array}\right], B_{1}=\left[\begin{array}{c}
0 \\
0 \\
0 \\
\frac{k_{t}}{m_{u s}}
\end{array}\right], \\
B_{2}=\left[\begin{array}{cccc}
0 & \frac{-1}{m_{s}} & 0 & \frac{1}{m_{u s}}
\end{array}\right]^{T}, C=\left[\begin{array}{cccc}
1 & 0 & -1 & 0 \\
0 & 1 & 0 & -1 \\
1 & 0 & 0 & 0
\end{array}\right] \\
\Psi=-\left[\begin{array}{cccc}
0 & \frac{-1}{m_{s}} & 0 & \frac{1}{m_{u s}}
\end{array}\right]^{T}\left(c_{0} \dot{z}_{d e f}+u\right) .
\end{gathered}
$$

Since the multiplicative fault $\alpha$ is slowly varying or constant, the fault estimation can be considered as a problem of parameter estimation. This problem can be solved based on an adaptive observer for joint state-parameter estimation [17]. However, the effect of the disturbances is not taken into account explicitly. Moreover, regarding to vertical vehicle dynamics, the system is always affected by the unknown road disturbances. Therefore, the developements in [17] are adapted to our problem and applied for the automotive applications. An $H_{\infty}$ performance is here proposed to attenuate the disturbance effects on the state and fault estimation.

Let us consider now system (16) with multiplicative fault $\alpha$. It is assumed that the matrix pair $(A, C)$ in (16) is observable. Following [17], an adaptive observer of the following structure can be proposed to estimate both states and fault $\alpha$ :

$$
\left\{\begin{array}{l}
\dot{\Upsilon}(t)=(A-K C) \Upsilon(t)+\Psi(y, u, t) \\
\dot{\hat{x}}=A \hat{x}+B_{2} u+\Psi(y, u, t) \hat{\alpha}+K(y-C \hat{x})+\Upsilon(t) \dot{\hat{\alpha}} \\
\dot{\hat{\alpha}}=\Gamma \Upsilon^{\prime} C^{\prime} \Sigma(y-C \hat{x})
\end{array}\right.
$$

where $K$ is the adaptive observer gain to be determined. $\Gamma, \Sigma$ are some bounded symmetric positive-definite matrices to be tuned. Since the quarter car model is a LTI system, $K, \Gamma, \Sigma$ are considered constant. $\Upsilon(t)$ satisfies the following assumption:

Assumption 1: $\Upsilon \in R^{n} \times R^{p}$ is a matrix of signals generated by the ordinary differential equation (ODE) system

$$
\dot{\Upsilon}(t)=[A-K C] \Upsilon(t)+\Psi(t)
$$

Morever, to apply the adaptive observer approach, an important required condition is that $\Psi(y, u, t)$ is persistently exciting. The following lemma is used to get the convergence of the adaptive observer.

Lemma 1: [17] Assume that $\Psi(t)$ is persistently exciting, so that there exist positive constants $\alpha, \beta, T$ and some bounded symmetric positive definitve matrix $\Sigma \in R^{m} \times R^{m}$ such that, for all $t$, the following inequalities hold:

$$
\alpha I \leq \int_{t}^{t+T} \Upsilon^{T}(\tau) C^{T}(\tau) \Sigma(\tau) C(\tau) \Upsilon(\tau) d \tau \leq \beta I
$$

then the system: $\dot{z}(t)=-\Gamma \Upsilon^{\prime} C^{\prime} \Sigma C \Upsilon z(t)$ is exponentially stable

Let us denote $e_{x}(t)=x(t)-\hat{x}(t), e_{\alpha}(t)=\alpha(t)-\hat{\alpha}(t)$ the state and fault estimation errors respectively.

Since $\alpha$ is constant or slowly varying, then $\dot{\alpha} \simeq 0$, then (see [17]):

$$
\left\{\begin{array}{l}
e_{\alpha}=\alpha-\dot{\alpha}=-\dot{\hat{\alpha}} \\
\dot{e}_{x}=\dot{x}-\dot{\hat{x}}=(A-K C) e_{x}+\Psi(y, u) e_{\alpha}+B_{1} w-\Upsilon \dot{\hat{\alpha}}
\end{array}\right.
$$

Let us now define $\eta$ as a combination of state and fault estimation errors, i.e:

$$
\begin{aligned}
\eta & =e_{x}-\Upsilon e_{\alpha}, \\
e_{x} & =\eta+\Upsilon e_{\alpha}=\eta-\Upsilon \dot{\hat{\alpha}}
\end{aligned}
$$

then:

$$
\begin{gathered}
\dot{\eta}=(A-K C) \eta+B_{1} w \\
\dot{e}_{\alpha}=-\Gamma \Upsilon^{\prime} C^{\prime} \Sigma C \eta-\Gamma \Upsilon^{\prime} C^{\prime} \Sigma C \Upsilon e_{\alpha}
\end{gathered}
$$

As seen in the above equations, the estimation errors $e_{x}, e_{\alpha}$ are affected by the unknown road disturbance $w(t)$. Then, the disturbance effect has to be minimized. It is worth noting that from the Lemma 1, the homogenous term of (22), i.e $\dot{e}_{\alpha}=-\Gamma \Upsilon^{\prime} C^{\prime} \Sigma C \Upsilon e_{\alpha}$ is exponentially stable. Therefore, if $\eta(t) \longrightarrow 0$, then $e_{\alpha} \longrightarrow 0$, and $e_{x}=\eta+\Upsilon e_{\alpha} \longrightarrow 0$ provided that $\Upsilon$ is bounded. However, due to the presence of the road disturbance $w$, in this work, we aim at minimizing the effect of $w$ on the estimation errors $e_{x}, e_{\alpha}$. To this aim, the effect of $w$ on $\eta$ in (21) is minimized as follows:

Proposition 1: If there exists a matrix $K \in R^{n \times m}$ so that the system:

$$
\dot{\eta}(t)=[A-K C] \eta(t)+B_{1} w(t)
$$

satisfies the following conditions:

- when $w(t) \equiv 0$, the system is exponentially stable

- when $w(t) \neq 0$, the following $L_{2}$-induced gain performance criterion is satisfied :

$$
\min \quad \gamma \quad \text { s.t }\|\eta\|_{2}<\gamma\|w\|_{2}
$$

where $\|.\|_{2}$ stands for $\mathrm{L}_{2}$ norm.

Or equivalenty, if there exist a matrix $Y$ such that

$$
\left[\begin{array}{ccc}
A^{\prime} P+P A-C^{\prime} Y^{\prime}-Y C & P B_{1} & I \\
\star & -\gamma^{2} I & 0 \\
\star & \star & -I
\end{array}\right]<0
$$


then state and fault estimation can be performed using the adaptive observer (17), and the gain $K$ is given by $K=P^{-1} Y$.

Proof: Let us consider a candidate Lyapunov function $V(\eta)=\eta^{T} P \eta$ for the system (23). In order to satisfy the condition in (24), then the following inequality should be ensured:

$$
\dot{V}+\eta^{T} \eta-\gamma^{2} w^{T} w<0
$$

By applying The Bounded Real Lemma, it leads to:

$$
\left[\begin{array}{ccc}
(A-K C)^{\prime} P+P(A-K C) & P B_{1} & I \\
\star & -\gamma^{2} I & 0 \\
\star & \star & -I
\end{array}\right]<0
$$

Let $Y=P K$, one obtains (25).

Finally, the observer design problem consists in solving the following optimization problem:

$$
\min \gamma \text { s.t } P>0 \text { and (25) holds }
$$

Then the observer gain $K$ is computed by: $K=P^{-1} Y$, and the damper fault is given by $\hat{\alpha} \square$.

\section{Method 3: A SWitched LPV OBSERVER APPROACH}

This section briefly presents the method, presented in [18], to estimate an actuator fault using a switched LPV observer. This method is applied here for the first time to damper fault estimation. For this method, we consider directly the total damper force as the control input $u(t)$, i.e: $u(t)=F_{s a}(t)$. Let us now reconsider faulty damper force:

$$
\bar{F}_{s a}(t)=(1-\alpha) F_{s a}(t)=\lambda F_{s a}(t)=\lambda u(t) \quad(\lambda=1-\alpha)
$$

Then under the damper fault, the state space representation of the quarter vehicle model (1) is given as follows:

$$
\begin{aligned}
\dot{x}(t) & =A x(t)+B_{2} \lambda u(t)+B_{1} w(t) \\
y(t) & =C x(t)
\end{aligned}
$$

$x=\left(z_{s}, \dot{z}_{s}, z_{u s}, \dot{z}_{s}\right)^{T} \in R^{n}$ is the state vector, $w=z_{r}$ is the disturbance input, $u \in R^{m}$ is the control input.

$$
\begin{aligned}
& A=\left[\begin{array}{cccc}
0 & 1 & 0 & 0 \\
\frac{-k_{s}}{m_{s}} & 0 & \frac{k_{s}}{m_{s}} & 0 \\
0 & 0 & 0 & 1 \\
\frac{k_{s}}{m_{u s}} & 0 & -\frac{k_{s}+k_{t}}{m_{u s}} & 0
\end{array}\right], B_{1}=\left[\begin{array}{c}
0 \\
0 \\
0 \\
\frac{k_{t}}{m_{u s}}
\end{array}\right], \\
& B_{2}=\left[\begin{array}{llll}
0 & \frac{-1}{m_{s}} & 0 & \frac{1}{m_{u s}}
\end{array}\right]^{T}, C=\left[\begin{array}{cccc}
1 & 0 & -1 & 0 \\
0 & 1 & 0 & -1 \\
1 & 0 & 0 & 0
\end{array}\right]
\end{aligned}
$$

Let consider now the faulty damper system (30), it can be seen that if the control input $u(t)=0$, then the damper fault information $\lambda$ in (30) becomes unobservable. It may cause the infeasibility problem in the observer design step when the control input equals zero. Thus, an interesting remedy, proposed in [18], is to consider a switched model depending on the sign of $u(t)$ in the observer synthesis step. Moreover, since the control input $u(t)$ is known, the system (30) can be represented as an LPV system by choosing $u(t)$ as a scheduling parameter. Let us rewrite $u(t)=|u(t)| \operatorname{sign}(u(t))$, and denote $\rho(t)=|u(t)|$ as a scheduling parameter. Then,

$$
u(t)=\rho(t) \operatorname{sign}(u(t))=\left\{\begin{array}{lll}
\rho(t) & \text { if } & u(t)) \geq 0 \\
-\rho(t) & \text { if } & u(t)<0
\end{array}\right.
$$

The scheduling parameter $\rho$ is assumed to satisfy: $\varepsilon \leq \rho \leq$ $\rho_{m}$, where $\varepsilon>0, \rho_{m}=u_{m}$ is the bound of the parameter $\rho$. The system (30) is now rewritten as the following LPV system:

$$
\dot{x}(t)=A x(t)+B_{\sigma}(\rho) \lambda+B_{1} w(t)
$$

where $B_{\sigma}(\rho)=B_{2} \rho \operatorname{sign}(u(t))$.

The original system (30) can be augmented in the following form:

$$
\begin{aligned}
{\left[\begin{array}{l}
\dot{x} \\
\dot{\lambda}
\end{array}\right] } & =\underbrace{\left[\begin{array}{cc}
A & B_{\sigma}(\rho) \\
0 & 0
\end{array}\right]}_{A_{e}(\rho)}\left[\begin{array}{l}
x \\
\lambda
\end{array}\right]+\left[\begin{array}{c}
B_{1} \\
0
\end{array}\right] w \\
y & =\underbrace{\left[\begin{array}{ll}
C & 0
\end{array}\right]}_{C_{e}}\left[\begin{array}{c}
x \\
\lambda
\end{array}\right]
\end{aligned}
$$

The system (33) is actually a switched system where $\sigma(t)$ is the switching rule that depends on the value of the function $\operatorname{sign}(u(t))$. Actually, one has $B_{\sigma}(\rho) \in\left\{-B_{2} \rho, B_{2} \rho\right\}$. Thus, the system (33) can be rewritten in the following form of a switched LPV system:

$$
\begin{aligned}
{\left[\begin{array}{l}
\dot{x} \\
\dot{\lambda}
\end{array}\right] } & =A_{e, \sigma}(\rho)\left[\begin{array}{l}
x \\
\lambda
\end{array}\right]+\left[\begin{array}{c}
B_{1} \\
0
\end{array}\right] w \\
y & =C_{e}\left[\begin{array}{l}
x \\
\lambda
\end{array}\right]
\end{aligned}
$$

where $A_{e, \sigma}(\rho) \quad$ switches among 2 subsystems $\left\{\left[\begin{array}{cc}A & -B_{2} \rho \\ 0 & 0\end{array}\right],\left[\begin{array}{cc}A & B_{2} \rho \\ 0 & 0\end{array}\right]\right\}$.

Consider the switched LPV system (34), the following switched LPV extended observer is proposed to estimate the system's state and the effectiveness coefficient $\lambda$ :

$$
\begin{aligned}
{\left[\begin{array}{l}
\dot{\hat{x}} \\
\dot{\hat{\lambda}}
\end{array}\right] } & =A_{e, \sigma}(\rho)\left[\begin{array}{l}
\hat{x} \\
\hat{\lambda}
\end{array}\right]+K_{\sigma}(t)(y-\hat{y}) \\
\hat{y} & =C_{e}\left[\begin{array}{l}
\hat{x} \\
\hat{\lambda}
\end{array}\right]
\end{aligned}
$$

From (34) and (35), the estimation error $e(t)$ is calculated by:

$\dot{e}=\left[\begin{array}{c}\dot{e}_{x} \\ \dot{e}_{\lambda}\end{array}\right]=A_{e, \sigma}(\rho)\left[\begin{array}{c}e_{x} \\ e_{\lambda}\end{array}\right]-K_{\sigma}(t)(y-\hat{y})+\left[\begin{array}{c}B_{1} \\ 0\end{array}\right] w$

Or equivalently:

$$
\dot{e}=\left[\begin{array}{c}
\dot{e}_{x} \\
\dot{e}_{\lambda}
\end{array}\right]=\left(A_{e, \sigma}(\rho)-K_{\sigma}(t) C_{e}\right)\left[\begin{array}{c}
e_{x} \\
e_{\lambda}
\end{array}\right]+B_{1 e} w
$$

where $B_{1 e}=\left[\begin{array}{c}B_{1} \\ 0\end{array}\right]$ and $K_{\sigma}(t)$ is the observer gain which has to be determined. The design of the LPV observer (35) is based also on the $H_{\infty}$ performance while one aims at minimizing the effect of the disturbances $w$ on the estimation error. The following theorem (whose complete proof is in [18]) allows to solve this problem:

Theorem 2: [18] Consider the switched system (34) and the switched extended observer (35), if there exists a collection of matrices $P_{i, k}>0, Y_{i, k}, k=0, \ldots K, \quad i=1,2$, of 
appropriate dimensions and $K$ is prescribed integer, such that for all $i=1,2$ and $j=1,2$, the following LMIs hold:

$$
\left[\begin{array}{ccc}
\frac{\left(P_{i, k+1}-P_{i, k}\right)}{T / K}+H e\left[P_{i, h} A^{(j)} e, \sigma-Y_{i, h} C_{e}\right] & \star & \star \\
B_{1 e}^{\prime} P_{i, h} & -\gamma^{2} I & \star \\
I & 0 & -I
\end{array}\right]<0
$$

for $k=0, \ldots K-1, h=k, k+1$,

$$
\begin{aligned}
& {\left[\begin{array}{ccc}
A^{(j)}{ }_{e, \sigma}^{\prime} P_{i, K}-C_{e}^{\prime} Y_{i, K}^{\prime}+P_{i, K} A^{(j)} e, \sigma-Y_{i, K} C_{e} & \star & \star \\
B_{1 e}^{\prime} P_{i, K} & -\gamma^{2} I & \star \\
I & 0 & -I
\end{array}\right]<0} \\
& P_{i, K}-P_{l, 0} \geq 0 \quad \forall l=1,2 \quad \& \quad l \neq i .
\end{aligned}
$$

then

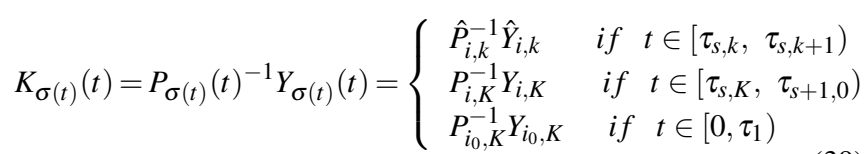

is the gain of the extended observer (35) and the error estimation asymptotically converges to zero for a dwell time of $T$, where

$Y_{\sigma(t)}(t)=\left\{\begin{array}{lr}Y_{i, k}+\left(Y_{i, k+1}-Y_{i, k}\right) \frac{t-\tau_{s, k}}{T / K}:=\hat{Y}_{i, k}, & \text { if } t \in\left[\tau_{s, k}, \tau_{s, k+1}\right) \\ Y_{i, K} & \text { if } t \in\left[\tau_{s, K}, \tau_{s+1,0}\right) \\ Y_{i_{0}, K} & \text { if } t \in\left[0, \tau_{1}\right)\end{array}\right.$

By designing the switched LPV observer, one obtain the estimation of $\lambda$, and the damper fault estimation is given by: $\hat{\alpha}=1-\hat{\lambda}$.

\section{Simulation Results}

The physical parameters characterize the considered quarter car model is given in the Table I.

\begin{tabular}{llllll}
\hline Parameter & $m_{S}[\mathrm{~kg}]$ & $m_{u s}[\mathrm{~kg}]$ & $k_{S}[\mathrm{~N} / \mathrm{m}]$ & $k_{t}[\mathrm{~N} / \mathrm{m}]$ & $\mathrm{c}[\mathrm{Nm} / \mathrm{s}]$ \\
Value & 315 & 37.5 & 29500 & 210000 & $\{700,5000\}$ \\
\hline
\end{tabular}

TABLE I

Parameters of Renault MÉGane Coupé Quarter CAR MOdel

The following scenario is used to test the performance of three different observers. It is assumed that the vehicle runs at $30 \mathrm{~km} / \mathrm{h}$ over a sinusoid road profile $w(t)=0.03 \sin (2 \pi t)$. Then, the nominal semi-active damper force is given by: $F_{s a}=c \dot{z}_{\text {def }}$ (blue line in Fig. 2), where $c$ is controlled damping of semi-active damper, satisfies $700 \leq c \leq 5000$.

The vehicle has a faulty semi-active damper because of a oil leakage. In the detail, from $t=0-5 \mathrm{~s}$, the damper looses $20 \%$ of its total force $(\alpha=0.2)$. From $t=5-8 \mathrm{~s}$, an higher fault occurs and the damper looses $60 \%$ of its efficiency $(\alpha=0.6)$.

It is noted that all observers are activated from $t=0.6 \mathrm{~s}$.

Fig. 2 plots the behavior of the nominal damper force $F_{s a}$ (blue liine), the faulty damper force $\bar{F}_{s a}\left(=(1-\alpha) F_{s a}=\right.$ $\left.F_{s a}+f\right)$ (green line) and the estimation of the additive fault $\hat{f}(t)$ (red line).

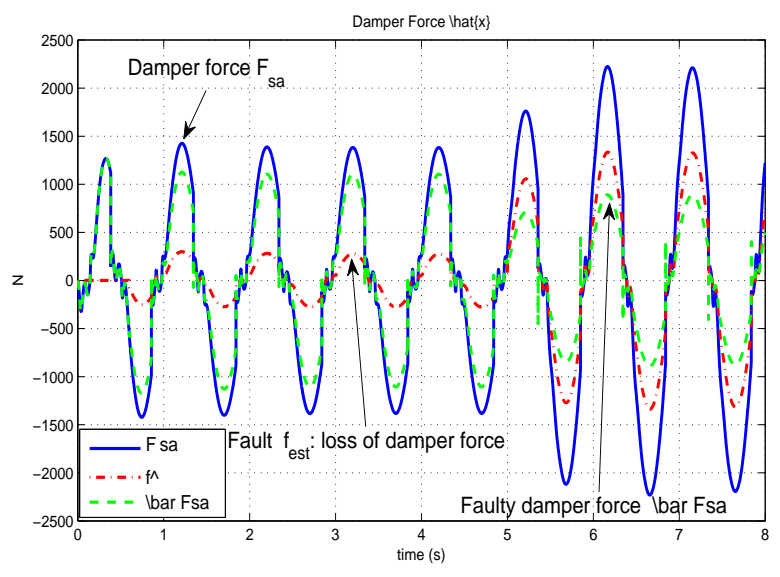

Fig. 2. Damper force behaviors
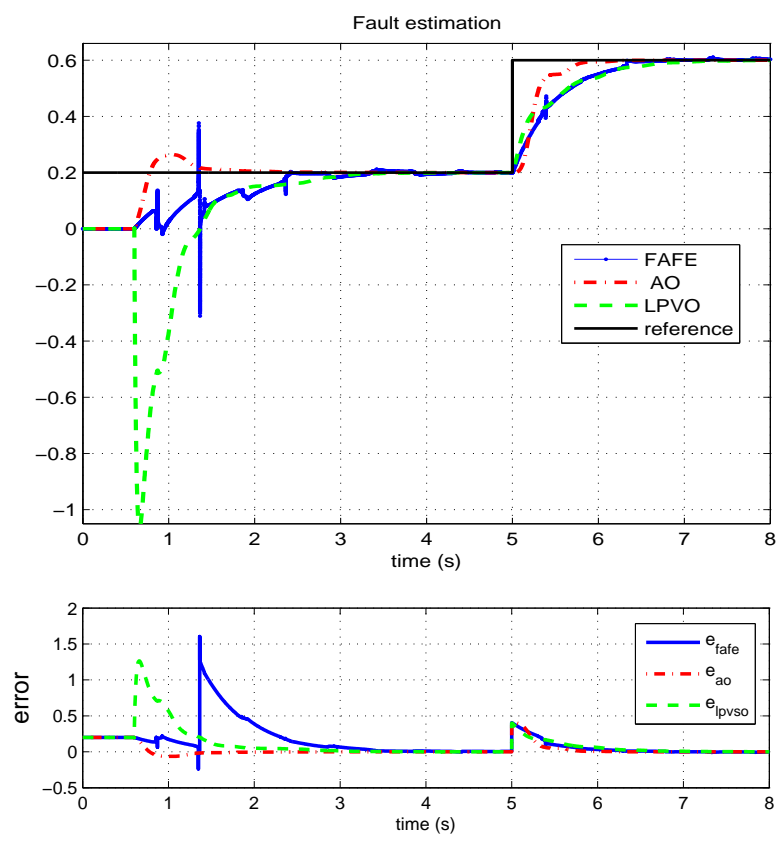

Fig. 3. Damper fault $\alpha$ estimation

Fig. 3 shows the comparison of the damper fault estimation using 3 different approaches: fast adaptive fault estimation approach (FAFE), Adaptive Observer approach (AO) and switched LPV observer approach (LPVO). The AO approach gives the estimation of $\alpha$. For the FAFE approach, the additive faut $f(t)$ is estimated and for LPVO approach, $\lambda$ is estimated. Then, the corresponding $\alpha$ is computed from $f(t), \lambda$ by $\hat{\alpha}=\hat{f} / F_{s a}$ or $\hat{\alpha}=1-\hat{\lambda}$. It can be seen from the estimation results that, despite of the presence of road disturbance, the estimation errors still converge to zeros. All three methods give a good estimation of damper fault. Moreover, it seems that the $\mathrm{AO}$ approach provides a better estimation than LPVO and FAFE approaches.

Now, let us consider the second scenario. It is assumed that the damper oil leak slowly from $t=0-6 \mathrm{~s}$, one has a loss of efficiency from $0 \rightarrow 80 \%$. 


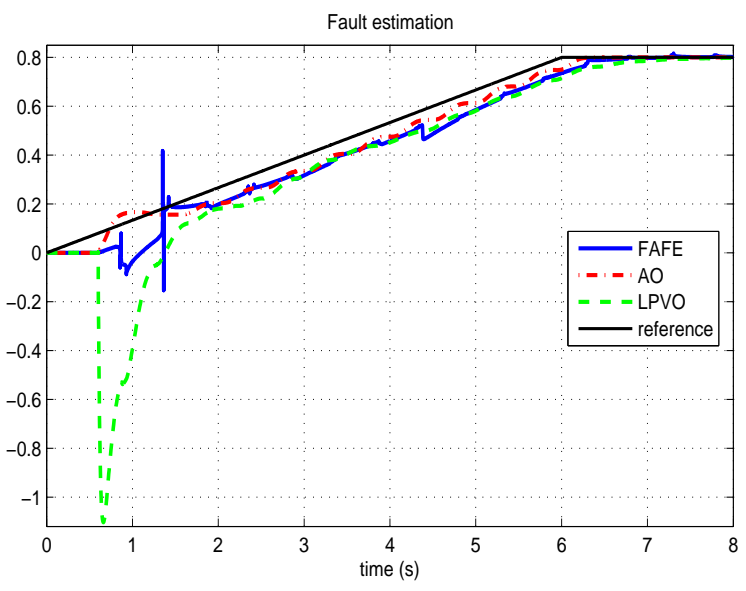

Fig. 4. Fault estimation $\alpha$ scenario 2

Fig. 4 demonstrates once again that the damper fault can be well estimated using the different observer approaches.

\section{CONCLUSIONS}

The paper provides a comparison of different methods for actuator fault estimation in semi-active suspension systems using FAFE observer, AO approach and a switched LPV observer approach. The damper fault is firstly modeled in different ways: additive model or multiplicative model. An $H_{\infty}$ performance is used to attenuate the effect of the road disturbance on the estimation error. The simulation results show that all three methods can give a good estimation results for damper fault. For the future work, fault tolerant control can be designed thanks to the fault estimation, and the implementation on a real testbed will be done.

\section{REFERENCES}

[1] J. Gertler, "Fault detection and isolation using parity relations," Control engineering practice, vol. 5, no. 5, pp. 653-661, 1997.

[2] S. Varrier, "Détection de situations critiques et commande robuste tolérante aux défauts pour l'automobile," $\mathrm{Ph} . \mathrm{D}$. dissertation, Institut National Polytechnique de Grenoble-INPG, 2013.

[3] C. Edwards, S. K. Spurgeon, and R. J. Patton, "Sliding mode observers for fault detection and isolation," Automatica, vol. 36, no. 4, pp. 541$553,2000$.

[4] F. Shi and R. Patton, "Active fault tolerant control of lpv descriptor systems based on extended state observers," in American Control Conference (ACC), 2014. IEEE, 2014, pp. 4458-4463.

[5] K. Zhang, B. Jiang, V. Cocquempot, et al., "Adaptive observer-based fast fault estimation," International Journal of Control Automation and Systems, vol. 6, no. 3, p. 320, 2008.

[6] M. Rodrigues, H. Hamdi, D. Theilliol, C. Mechmeche, and N. BenHadj Braiek, "Actuator fault estimation based adaptive polytopic observer for a class of lpv descriptor systems," International Journal of Robust and Nonlinear Control, 2014.

[7] A. Abdullah and M. Zribi, "Sensor-fault-tolerant control for a class of linear parameter varying systems with practical examples," Industrial Electronics, IEEE Transactions on, vol. 60, no. 11, pp. 5239-5251, 2013.

[8] R. J. Patton, L. Chen, and S. Klinkhieo, "An lpv pole-placement approach to friction compensation as an ftc problem," Int. J. Appl. Math. Comput. Sci., vol. 22, no. 1, pp. 149-160, March 2012.

[9] S. Montes de Oca, V. Puig, and J. Blesa, "Robust fault detection based on adaptive threshold generation using interval lpv observers," International Journal of Adaptive Control and Signal Processing, vol. 26, no. 3, pp. 258-283, 2012. [Online]. Available: http://dx.doi.org/10.1002/acs.1263
[10] M. Hamayun, H. Alwi, and C. Edwards, "An lpv fault tolerant control scheme using integral sliding modes," in Decision and Control (CDC), 2012 IEEE 51st Annual Conference on, 2012, pp. 1840-1845.

[11] S. Montes de Oca, D. Rotondo, F. Nejjari, and V. Puig, "Fault estimation and virtual sensor ftc approach for lpv systems," in Decision and Control and European Control Conference (CDC-ECC), 2011 50th IEEE Conference on, 2011, pp. 2251-2256.

[12] D. Fischer and R. Isermann, "Mechatronic semi-active and active vehicle suspensions," Control Engineering Practice, vol. 12, no. 11, pp. 1353-1367, 2004.

[13] C. Poussot-Vassal, C. Spelta, O. Sename, S. M. Savaresi, and L. Dugard, "Survey and performance evaluation on some automotive semi-active suspension control methods: A comparative study on a single-corner model," Annual Reviews in Control, vol. 36, no. 1, pp. 148-160, 2012.

[14] O. Sename, J.-C. Tudon-Martinez, and S. Fergani, "Lpv methods for fault-tolerant vehicle dynamic control," in Control and Fault-Tolerant Systems (SysTol), 2013 Conference on. IEEE, 2013, pp. 116-130.

[15] M. Q. Nguyen, O. Sename, and L. Dugard, "An lpv fault tolerant control for semi-active suspension-scheduled by fault estimation," IFAC-PapersOnLine, vol. 48, no. 21, pp. 42-47, 2015.

[16] D. Hernandez-Alcantara, R. Morales-Menendez, L. AmezquitaBrooks, O. Sename, and L. Dugard, "Fault estimation methods for semi-active suspension systems," in 17th IEEE International Autumn Meeting on Power, Electronics and Computing (ROPEC'2015), 2015.

[17] Q. Zhang, "Adaptive observer for multiple-input-multiple-output (mimo) linear time-varying systems," Automatic Control, IEEE Transactions on, vol. 47, no. 3, pp. 525-529, 2002.

[18] M. Q. Nguyen, O. Sename, and L. Dugard, "A switched lpv observer for actuator fault estimation," IFAC-PapersOnLine, vol. 48, no. 26, pp. 194-199, 2015. 